\title{
Influencia de la temperatura ambiental sobre la presión arterial del perro
}

\author{
Cainzos, R.P.; Koscinczuk, P.; Ferreiro, M.C. \\ Cátedra de Patología Médica, Facultad de Ciencias Veterinarias, Universidad Nacional del Nordeste, \\ Sargento Cabral 2139 (3400), Corrientes, Argentina. \\ romicainzos@hotmail.com
}

\begin{abstract}
Resumen
Cainzos, R.P.; Koscinczuk, P.; Ferreiro, M.C.: Influencia de la temperatura ambiental sobre la presión arterial del perro. Rev. vet. 25: 2, 154-157, 2014. En los animales domésticos el mantenimiento de la temperatura corporal dentro de límites normales supone la activación de numerosas respuestas fisiológicas que generan variaciones en el tono vascular y la presión arterial. El objetivo de este trabajo fue evaluar la influencia de la temperatura ambiental sobre la presión arterial en perros sanos y conscientes (no sedados), en ambientes con temperaturas superiores a los $38^{\circ} \mathrm{C}$ versus climatizados a $24^{\circ} \mathrm{C}$. Para ello se utilizó el método oscilométrico (monitor de signos vitales V6Vet ${ }^{\circledR}$ ), registrándose las presiones sistólica, diastólica y media (PS, PD, PM) en el miembro anterior derecho. Cuando la temperatura fue superior a $38^{\circ} \mathrm{C}$, tanto PS como PD y PM registraron los promedios más altos (136, 80 y 96 $\mathrm{mmHg}$ respectivamente); en cambio con temperaturas de $24^{\circ} \mathrm{C}$ fueron de 123,68 y $91 \mathrm{mmHg}$. Únicamente PS arrojó diferencia significativa $(\mathrm{p}<0,05)$. Si bien los aumentos de temperatura ambiental afectan las presiones sanguíneas, el cambio más importante es la elevación de PS.
\end{abstract}

Palabras clave: perro, presión arterial, temperatura ambiental.

\begin{abstract}
Cainzos, R.P.; Koscinczuk, P.; Ferreiro, M.C.: Influence of environmental temperature on the canine arterial pressure. Rev. vet. 25: 2, 154-157, 2014. In domestic animals the maintenance of the internal temperature within normal range is the result of the activation of many physiological responses that change vascular tone and blood pressure. The aim of this study was to evaluate arterial pressure in conscious dogs (not sedated) in an environment with temperature above $38^{\circ} \mathrm{C}$ and other with temperature of $24^{\circ} \mathrm{C}$. For measurements of blood pressure, oscillometric method was used (vital signs monitor V6Vet $\left.{ }^{\circledR}\right)$. The recorded variables were: systolic, diastolic and medium pressures (SP, DP, MP) in the right fore limb. When the temperature was above $38^{\circ} \mathrm{C}, \mathrm{SP}, \mathrm{DP}$ and MP registered the higher means $(136,80$ and $96 \mathrm{mmHg}$, respectively). When the temperature was $24^{\circ} \mathrm{C}$ values were 123,68 and 91 $\mathrm{mmHg}$, respectively. Only SP registered significant difference $(\mathrm{p}<0.05)$. Although adverse climatic condition could affect blood pressure, SP could reflect those changes in a consistent way.
\end{abstract}

Key words: dog, arterial pressure, environmental temperature.

\section{INTRODUCCIÓN}

La presión sanguínea arterial sistémica es uno de los parámetros cardiovasculares que se utiliza para controlar el estado hemodinámico de los pacientes. La presión se determina como el producto del gasto cardiaco por la resistencia vascular periférica, y es controlada por un complejo sistema neuronal, altamente integrado con mecanismos endocrinos y paracrinos, para mantener un nivel tal que asegure la adecuada perfusión

Recibido: 7 mayo 2014 / Aceptado: 12 junio 2014 tisular y oxigenación de todo el organismo, incluso en las situaciones más exigentes ${ }^{10}$.

El sistema nervioso autónomo, través de sus ramas simpática y parasimpática, genera respuestas inmediatas a la exposición de agentes estresantes ${ }^{9}$, como los cambios importantes de temperatura ambiental, induciendo modificaciones del estado fisiológico con un incrementos de frecuencia cardiaca y presión arterial ${ }^{4}$. Casi simultáneamente, las fibras simpáticas estimulan a la médula adrenal para que libere adrenalina.

El incremento de los niveles circulantes de adrenalina (primariamente desde la médula adrenal) y de 
noradrenalina (de los nervios simpáticos), amplía las respuestas cardio-circulatorias: frecuencia cardiaca, fuerza de contracción y vasoconstricción periférica ${ }^{1}$. La excitación del sistema simpático se desvanece rápidamente a consecuencia de la activación del sistema parasimpático. Esta modulación morigera el tono vagal en corazón y pulmones, en el intento de reducir los efectos deletéreos de la hipertensión ${ }^{4}$.

Se reconoce que las temperaturas extremas -mucho frío o excesivo calor- pueden asociarse a estrés térmico. Los centros supra y preóptico del cerebro son los encargados del control térmico; sus células nerviosas responden tanto al calentamiento (neuronas sensibles al calor) como al enfriamiento (neuronas sensibles al frío). La función de estos centros es coordinar la información sensorial recibida y enviar señales a la piel (vasoconstricción o vasodilatación periférica), así como a los músculos y a otros órganos implicados en la regulación térmica ${ }^{5}$. De la misma manera que en medicina humana se reconoce el efecto que las condiciones climáticas ejercen sobre la presión sanguínea, es de esperar que tanto la temperatura como la humedad, impacten sobre el sistema cardiocirculatorio de los caninos.

Cuando el organismo se enfrenta a condiciones térmicas que se alejan de la confortable (frío o calor), se ponen en marcha mecanismos para controlar la temperatura interna. Ante la pérdida de calor se produce vasoconstricción periférica, sobre todo en las extremidades, y la sangre se torna más densa reduciendo la disipación del calor por conducción. Al contrario, cuando la temperatura corporal aumenta $\left(39^{\circ} \mathrm{C}\right.$ en perros), se activan los mecanismos de la termólisis (jadeo y aumento del flujo sanguíneo periférico) para evitar complicaciones como el edema cerebral ${ }^{5}$.

Las glándulas sudoríparas asumen escasa importancia en la termorregulación del perro (su principal función es liberar feromonas). El mecanismo primordial para la pérdida de calor es la evaporación de agua por jadeo. A diferencia de lo que habitualmente se cree, los perros no se enfrían utilizando la superficie de la lengua, sino que lo hacen en mayor medida a través de los cornetes nasales ${ }^{6}$, cuya estructura compleja les

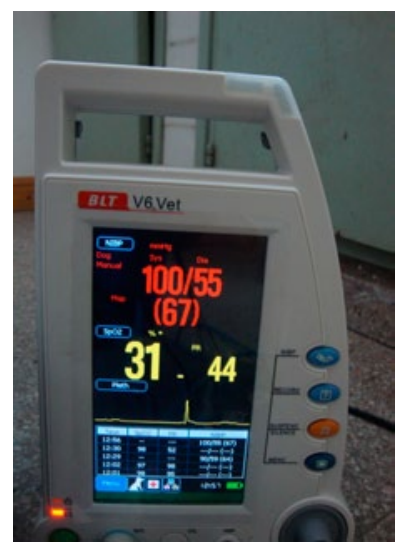

Figura 1. Monitor de signos vitales. La pantalla muestra las presiones sanguíneas, la saturación de oxígeno y el pulso. confiere una gran superficie, extremadamente vascularizada, que posibilita una rápida y eficaz pérdida de calor. Además los caninos poseen una glándula nasal lateral que coadyuva al enfriamiento por evaporación; por ello puede afirmarse que la nariz del perro desarrolla funciones respiratoria, olfativa y de termorregulación ${ }^{6}$. Por afectar la cantidad de agua corporal, los mecanismos encargados de mantener la neutralidad térmica son capaces de provocar variaciones de las presiones sanguíneas ${ }^{5}$.

El objetivo de este trabajo fue evaluar las modificaciones de la presión arterial en perros sanos y conscientes (no sedados), en ambientes con temperaturas superiores a $\operatorname{los} 38^{\circ} \mathrm{C}$ y en ambientes climatizados a $24^{\circ} \mathrm{C}$.

\section{MATERIAL Y MÉTODOS}

De los animales. Se utilizaron trece perros de las razas Beagle, Fox Terrier Smooth y Wire, de la ciudad de Corrientes. Los animales eran clínicamente sanos, adultos entre dos y ocho años de edad, de ambos sexos. Se destaca que, al momento del primer registro, los animales no estaban familiarizados con los investigadores ni con el procedimiento de medición de la presión arterial.

Del diseño del estudio. Cada canino recibió dos tratamientos: a) evaluación de la presión arterial al aire libre con temperatura ambiente superior a $38^{\circ} \mathrm{C}$ y b) evaluación de la presión arterial en un ambiente climatizado con temperatura de $24^{\circ} \mathrm{C}$.

Del registro de presión arterial. La medición de las variables presión sistólica (PS), presión diastólica (PD) y presión media (PM) se realizó mediante el método oscilométrico, utilizando un monitor de signos vitales de uso veterinario $\left(\mathrm{V} 6 \mathrm{Vet}^{\circledR}\right)$. La precisión del monitor oscilométrico es dependiente de su capacidad de encontrar dos pulsos emparejados dentro de un determinado período de tiempo (Figura 1).

En todos los casos, antes de realizar las mediciones los sujetos de estudio permanecieron en el lugar por 5

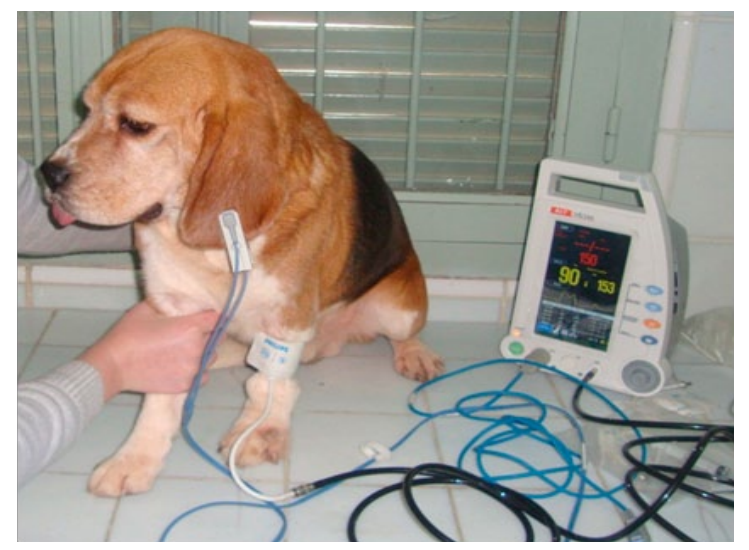

Figura 2. Toma de presión. Manguito colocado en el miembro anterior. 
Tabla 1. Valores de presión sistólica (PS), diastólica (PD) y media (PM) para ambas temperaturas ambientales.

\begin{tabular}{lccccc}
\hline presión & $24^{\circ} \mathrm{C}$ & $\mathrm{Rs}-\mathrm{Ri}$ & $>38^{\circ} \mathrm{C}$ & $\mathrm{Rs}-\mathrm{Ri}$ & $\mathrm{p}$ \\
\hline PS (mmHg) & 123 & $144-101$ & 136 & $159-112$ & $<0.0070$ \\
$\mathrm{PD}(\mathrm{mmHg})$ & 68 & $86-49$ & 80 & $103-56$ & $=0.1822$ \\
$\mathrm{PM}(\mathrm{mmHg})$ & 91 & $114-67$ & 96 & $119-72$ & $=0.3049$ \\
$\overline{\mathbf{x}}(\mathrm{mmHg})$ & 94 & - & 104 & - & - \\
\hline
\end{tabular}

Valores expresados en media aritmética, rango superior (Rs) e inferior (Ri); p: significancia; $\bar{x}$ : media.

a 10 minutos, para habituarse al entorno. La toma de la presión se llevó a cabo con el animal en estación o sentado. Un operador experimentado lo sujetó cuidadosamente (sin forcejear), mientras otro le colocaba el manguito en el miembro anterior derecho, en coincidencia con la arteria antibraquial (Figura 2). En cada caso, el manguito fue seleccionado teniendo en cuenta que fuera el $40 \%$ más grande que la circunferencia del miembro en los perros.

De la significación estadística de los resultados. Los valores obtenidos fueron volcados en una planilla de cálculo Excel, junto con los datos para identificar al animal (nombre, edad, sexo y raza). Los guarismos fueron tratados inicialmente con estadísticas descriptivas (media aritmética y rangos superior e inferior). La diferencia entre los valores obtenidos en sendos ambientes, se obtuvo por análisis no paramétrico de la variancia (Kruskal Wallis), considerando como único factor la temperatura ambiente. La significancia se fijó en 5\%, por debajo de la cual se rechazó la hipótesis nula de igualdad.

\section{RESULTADOS Y DISCUSIÓN}

Las presiones en todos los animales bajo estudio fueron más elevadas cuando la temperatura ambiente superó los $38^{\circ} \mathrm{C}$. En estos sujetos, el valor medio más alto para la sístole fue de $136 \mathrm{mmHg}$, siendo el promedio de presiones de $104 \mathrm{mmHg}$. En cambio, cuando la temperatura se mantuvo en $24^{\circ} \mathrm{C}$, el valor sistólico medio más alto fue de $123 \mathrm{mmHg}$ y el promedio de 94 mmHg (Tabla 1).

Con respecto a las presiones, los valores más altos registrados en ambos ambientes (temperatura superior a $38^{\circ} \mathrm{C}$ y ambiente controlado $24^{\circ} \mathrm{C}$ ) fueron los de la presión sistólica, siendo ésta la única diferencia estadísticamente significativa $(p<0,05)$. En cambio, las presiones diastólica y media no fueron significativas ( $>0,05)$ (Tabla 1).

Se ha reportado que la elevación de la presión arterial sistólica, aislada o en combinación con la diastólica, es comúnmente diagnosticada tanto en perros como en gatos, mientras que el hallazgo de hipertensión diastólica parece ser raro en animales domésticos ${ }^{3}$. El canino se considera hipertenso cuando la presión arterial es igual o superior a $160 \mathrm{mmHg}$; frecuentemente se acompaña por alteraciones del hemograma, el perfil bioquí- mico sérico y el urianálisis ${ }^{3}$. En los perros, la presión sanguínea tiende a elevarse con la edad, registrándose aumentos de 1-3 mmHg por año, a consecuencia de cambios relacionados con el envejecimiento ${ }^{8}$.

Además de las variaciones dentro del día y entre días, está comprobado que la presión arterial se eleva al visitar al veterinario, especialmente si los animales no están habituados a la medición ni al operador, lo que genera un estrés social ${ }^{7}$. Los perros sensibles al estrés presentan presiones más altas, así como frecuencias cardíacas más elevadas. La intensidad de los agentes estresantes aumenta la variabilidad de las respuestas, como sucede en ambientes externos con altas temperaturas ${ }^{10}$; por ello las mediciones deber ser efectuadas de manera precisa, sin disturbio alguno.

En la presente experiencia, las mediciones efectuadas en ambiente controlado fueron más rápidas, se realizaron con pocas personas en una habitación cerra$\mathrm{da}$, lejos de otros animales y sin ruidos de fondo. Se considera que el miedo y la ansiedad pueden ser contrarrestados por un lapso de relajación de 5-10 minutos, tras los cuales los sujetos se aclimatan al entorno ${ }^{3}$. El operador debe estar capacitado para efectuar una hábil manipulación de los animales y el equipo, según se trate del método oscilométrico o por efecto Doppler ${ }^{2,3}$.

El método oscilométrico aquí utilizado es considerado confiable cuando se aplica en animales de razas medianas y grandes. En cambio, se torna inseguro en animales pequeños, menores de $4,5 \mathrm{~kg}$, por lo cual no está recomendado su empleo en gatos ${ }^{2}$. La técnica es clínicamente aceptable por ser poco invasiva. Puede aplicarse en perros no sedados, pero las frecuencias cardíacas lentas y los movimientos de las extremidades disminuirán la precisión de los resultados. El método Doppler es de elección en animales pequeños y en pacientes anestesiados (ampliamente utilizado en cirugía veterinaria), aunque su confiabilidad se restringe a la presión sistólica, debido a la discriminación imprecisa de los sonidos correspondientes a la presión diastólica ${ }^{3,7}$.

La medición de la presión arterial en perros y gatos no es todavía una práctica de rutina en la clínica diaria, a pesar de ser una herramienta poco invasiva y de bajo costo. Su determinación es importante porque el diagnóstico temprano de hipertensión puede coadyuvar en el tratamiento de afecciones cardíacas, renales, endocrinas y neurológicas, así como la obesidad, entre otras. La hipertensión no solo puede contribuir a la insuficiencia cardiaca sino también aumentar la tensión en otros órganos, provocando daños estructurales y deterioro de sus funciones. En el otro extremo, la hipotensión ortostática, puede condicionar eventos sincopales e hipoperfusión de órganos con el consiguiente daño isquémico ${ }^{11}$.

Se concluye que los caninos registran presiones arteriales (sistólica, diastólica y media) más elevadas en 
ambientes cálidos y viceversa. En los exámenes clínicos, considerar la temperatura ambiente permitirá interpretar las mediciones con mayor precisión y lograr un diagnóstico más certero.

\section{REFERENCIAS}

1. Bergamasco L, Osella MC, Savarino P, Larosa G, Ozella L, Manassero M, Badino P, Odore R, Bardero R, Re G. 2010. Heart rate variability and saliva cortisol assessment in shelter dog: Human-animal interaction effects. Appl Anim Behaviour Sci 125: 56-68.

2. Brown S, Atkins C, Bagley R, Carr A, Cowgill L, Davidson M, Egner B, Elliot J, Henik R, Labato M, Littman M, Polzin D, Ross L, Snyder P, Stepien R. 2007. Guidelines for the identification, evaluation and management of systemic hypertension in dogs and cats. J Vet Intern Med 21: 542-558.

3. Henik RA, Dolson MK, Wenholz LJ. 2005. How to obtain a blood measurement. Clin Tech Small Anim Pract 20: 144-150.

4. Iversen S, Iversen L, Saper CB. 2000. The autonomic nervous system and the hypothalamus. In: Principles of neural science (Kandel ER, Schwartz JH \& Jessell TM ed.), McGraw-Hill, New York, p. 960-981.

5. Kenney LW. 1998. Enciclopedia de seguridad y salud en el trabajo, Ed. OIT-Agencia Española de Cooperación Internacional, Madrid, $75 \mathrm{p}$.
6. Oechtering G. 2010. Síndrome braquicefálico. Nuevos conocimientos sobre una vieja enfermedad congénita. Vet Focus 20: 2-9.

7. Rattez EP, Reynolds BS, Concordet D, Layssol CJ, Segalem MM, Chetboul V, Lefebvre HP. 2009. Withinday and between-day variability of blood pressure measurement in healthy conscious Beagle dogs using a new oscillometric device. $J$ Vet Cardiol 12: 35-40.

8. Rothwell PM, Howard SC, Dolan E, O'Brien E, Dobson JE, Dahlof B, Sever PS, Poulter NR. 2010. Prognostic significance of visit-to-visit variability, maximum systolic blood pressure, and episodic hypertension. Lancet 375: 895-905.

9. Ulrich-Lai YM, Herman JP. 2009. Neural regulation of endocrine and autonomic stress responses. Nat Rev Neurosci 10: 397-409.

10. Vincent IC, Michell AR. 1995. Relationship between blood pressure and stress-prone temperament in dogs. Physiol Behav 60: 135-138.

11. Ward G, Milliken P, Patel B, McMahon N. 2012. Comparison of non-invasive and implanted telemetric measurement of blood pressure and electrocardiogram in conscious beagle dogs. J Pharm Toxicol Meth 10: 1016. 